\title{
Synthesis of a novel D-A type photoelectrical material based on carbazole
}

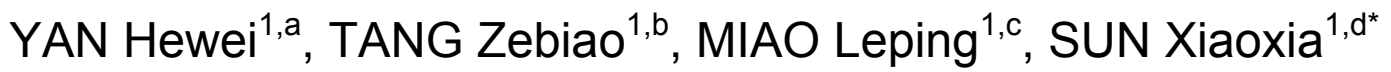 \\ ${ }^{1}$ Jiangxi Key Laboratory of Organic Chemistry, Jiangxi Science and Technology Normal University, \\ Nanchang 330013, People's Republic of China \\ ayanhewei1990@163.com; btangzebiao88@163.com; 'miaoleping@163.com; \\ sunxiaoxia77@126.com
}

Keywords: D-A type molecules; carbazole; Knevenagel reaction

\begin{abstract}
Aliphatic chains and cyanoacetic acid could improve the solubility and the optical and electrical properties of small molecules. Novel D-A type conjugated organic molecules composed of central carbazole units and cyanoacetic acid terminal groups have been designed and constructed. Optical properties, electrical Properties and thermodynamic properties will be studied in future work. The target compounds would be promising candidates for organic light-emitting diodes.
\end{abstract}

\section{Introduction}

The D-A type molecules have attracted a variety of attention during the past decade due to their great potential electronic and optoelectronic applications in photovoltaic cells, electrochromic cells and organic light-emitting diodes. ${ }^{1}$ Comparing with organic polymers, conjugated organic molecules have great advantages on higher purity, fixed molecular weight and their clear molecular structure, which will receive a significant amount of interest by researchers in the coming years. ${ }^{2}$ Especially, those with donor-acceptor (D-A) architectures, play an key role in organic materials leading to desirable semiconductor properties for electrical and opticalapplications.

Carbazole and its derivatives have many great properties such as rigid plane, long conjugation length, good hole transporting properties and charge transporting compounds creating free carriers in the visible region through the photo-carrier generation process. So those often been applied to biochemical fluorescent technology, electrogenerated chemiluminescence, organic light emitting diodes (OLEDs), and photovoltaic cells. ${ }^{3-5}$

Herein, we design and synthesize new compounds (2E,2'E)-3,3'-(5,5'-(9-octyl-9H -carbazole-3,6-diyl)bis(thiophene-5,2-diyl))bis(2- cyanoacrylic acid), based on carbazole units at the center and introduced cyanoacetic acid group and thiophene to improve electronic and optical properties of organic conjugated molecules. The target compounds would be promising candidates for organic light-emitting diodes. Optical properties, electrical Properties and thermodynamic properties will be studied in future work.

\section{Experimental Section}

Characterization: ${ }^{1} \mathrm{H}$ NMR and ${ }^{13} \mathrm{C}$ NMR spectra were collected on a Bruker AM-400WB spectrometer in chloroform-d or DMSO as solvent and tetramethylsilane (TMS) as the internal standard. Materials: Diethyl ether and tetrahydrofuran (THF) were dried over sodium benzophenone and distilled under argon atmosphere before use. $\mathrm{CHCl}_{3}$ and $\mathrm{DMF}$ were dried over $\mathrm{CaH}_{2}$ and then distilled under nitrogen atmosphere and deoxygenated by purging with nitrogen for 30 min before use in monomer synthesis. Other chemicals were purchased from Acros and used without further purification. 


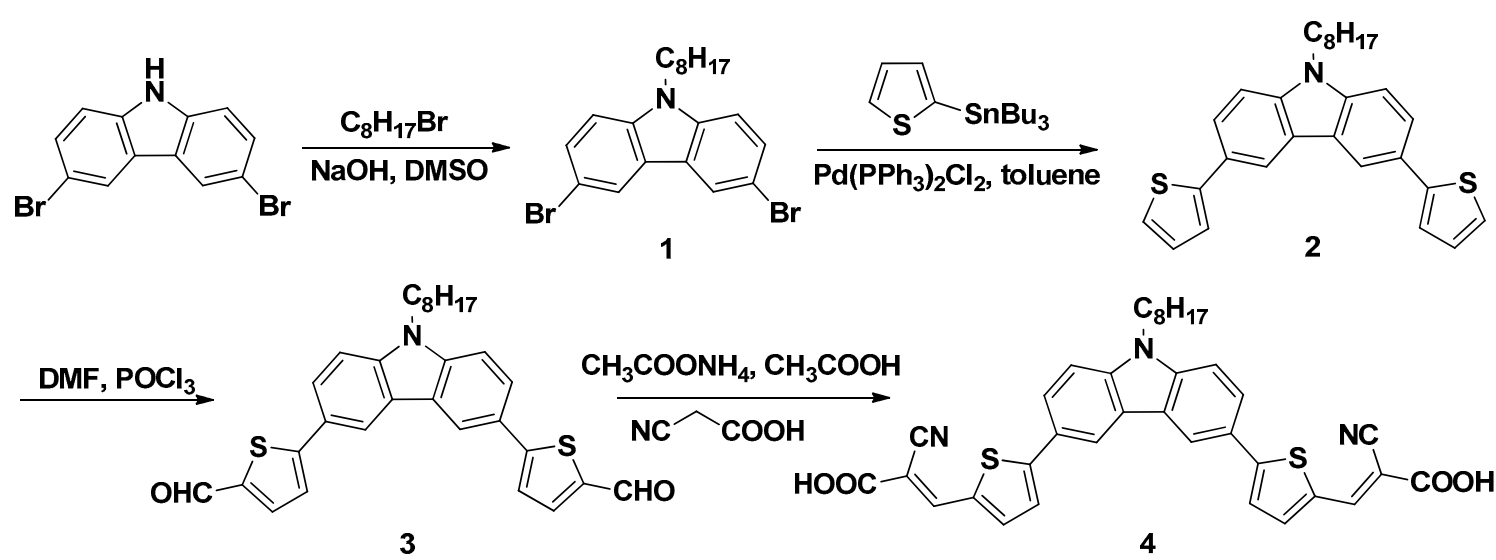

Scheme 1. The synthesis route of (2E,2'E)-3,3'-(5,5'-(9-octyl-9H-carbazole-3,6-diyl) bis(thiophene-5,2-diyl))bis(2- cyanoacrylic acid) (4)

In our previous paper, compounds $\mathbf{2}$ and $\mathbf{3}$ have been constructed via the Susuki coupling reaction and the Vilsmeier-Haack reaction, respectively, as shown in Scheme 1. Firstly, 3,6-dibromo -9H-carbazole and 1-bromooctane reacted in DMSO under a mild condition and simple purification process to give 1 in $86 \%$ yield. Then, 3,6-dibromo-9-octyl-9H-carbazole, 2.5 equiv. of tributyl (thiophen-2- yl)stannane and $\mathrm{Pd}\left(\mathrm{PPh}_{3}\right)_{2} \mathrm{Cl}_{2}$ used as a catalyst in toluene afforded 2 in a yield of $78 \%$ after multiple reaction route selections. At last, DMF and $\mathrm{POCl}_{3}$ were added to 2 to give 3 in $84 \%$ yi

Synthesis of 3,6-dibromo-9-octyl-9H-carbazole (1)

3,6-dibromo-9H-carbazole $(2.0 \mathrm{~g}, 6.15 \mathrm{mmol})$ and 1-bromooctane $(1.43 \mathrm{~g}, 7.38 \mathrm{mmol})$ were dissolved in DMSO $(40 \mathrm{ml})$ and stirred for $30 \mathrm{~min}$ at room temperature, A sodium hydroxide solution ( $5 \mathrm{ml}, \mathrm{v}: 50 \%$ ) was slowly added and then stirred for overnight at room temperature. Then the reaction mixture was poured into water. The precipitate was filtered, repeatedly washed with distilled water and dried under vacuum. The product was obtained as off-white solids without further purifications. Yield: $2.3 \mathrm{~g},(86 \%) .{ }^{1} \mathrm{H} N M R\left(400 \mathrm{MHz}, \mathrm{CDCl}_{3}, \mathrm{ppm}\right): \delta=8.08(\mathrm{~s}, 2 \mathrm{H}), 7.48(\mathrm{~d}, \mathrm{~J}=8.8$ $\mathrm{Hz}, 2 \mathrm{H}), 7.20(\mathrm{~d}, \mathrm{~J}=7.2 \mathrm{~Hz}, 2 \mathrm{H}), 4.16(\mathrm{t}, \mathrm{J}=6.8 \mathrm{~Hz}, 2 \mathrm{H}), 1.74(\mathrm{~d}, \mathrm{~J}=6.4 \mathrm{~Hz}, 2 \mathrm{H}), 1.23-1.15(\mathrm{~m}, 10 \mathrm{H})$, $0.79(\mathrm{t}, \mathrm{J}=6.4 \mathrm{~Hz}, 3 \mathrm{H}) .{ }^{13} \mathrm{C}$ NMR $\left(100 \mathrm{MHz}, \mathrm{CDCl}_{3}\right): \delta 138.00,127.76,122.27,111.03,109.01$, $42.06,30.69,28.15,27.72,26.11,21.54,13.04$.

Synthesis of 9-octyl-3,6-di(thiophen-2-yl)-9H-carbazole (2)

A mixture of 3,6-dibromo-9-octyl-9H-carbazole $(0.50 \mathrm{~g}, 1.14 \mathrm{mmol})$, tributyl(thiophen-2 -yl)stannane $(1.07 \mathrm{~g}, 2.86 \mathrm{mmol})$, and $\mathrm{Pd}\left(\mathrm{PPh}_{3}\right)_{2} \mathrm{Cl}_{2}(0.09 \mathrm{~g}, 0.13 \mathrm{mmol})$ in dry toluene $(30 \mathrm{ml})$ was stirred at $115{ }^{\circ} \mathrm{C}$ under $\mathrm{N}_{2}$ for $24 \mathrm{~h}$. After cooling the mixture to the room temperature, the solvent was directly removed, The crude product was purified using column chromatography on a silica gel with petroleum ether as the eluent. The product was obtained as white solid. Yield: $0.40 \mathrm{~g},(78 \%) .{ }^{1} \mathrm{H}$ NMR $\left(400 \mathrm{MHz}, \mathrm{CDCl}_{3}, \mathrm{ppm}\right): \delta=8.27(\mathrm{~s}, 2 \mathrm{H}), 7.66(\mathrm{~d}, \mathrm{~J}=8.0 \mathrm{~Hz}, 2 \mathrm{H}), 7.30(\mathrm{t}, \mathrm{J}=7.4 \mathrm{~Hz}, 4 \mathrm{H}), 7.19(\mathrm{~d}, \mathrm{~J}$ $=5.2 \mathrm{~Hz}, 2 \mathrm{H}), 7.04(\mathrm{~s}, 2 \mathrm{H}), 4.22(\mathrm{t}, \mathrm{J}=7.0 \mathrm{~Hz}, 2 \mathrm{H}), 1.81(\mathrm{t}, \mathrm{J}=6.6 \mathrm{~Hz}, 2 \mathrm{H}), 1.31-1.15(\mathrm{~m}, 10 \mathrm{H}), 0.80$ (m, 3H). ${ }^{13} \mathrm{C}$ NMR $\left(100 \mathrm{MHz}, \mathrm{CDCl}_{3}\right): \delta 144.54,139.21,127.06,124.70,123.37,122.52,122.03$, $120.94,116.90,108.15,42.01,30.72,28.30,27.89,26.96,21.55,13.05$.

\section{Synthesis of 5,5'-(9-octyl-9H-carbazole-3,6-diyl)bis(thiophene-2-carbaldehyde) (3)}

9-octyl-3,6-di(thiophen-2-yl)-9H-carbazole (0.84g, $1.89 \mathrm{mmol})$ and DMF (4.0 $\mathrm{ml})$ was dissolved in 1,2-dichloroehane $(30 \mathrm{ml})$ and Phosphorus oxychloride $(6 \mathrm{ml})$ was added dropwise, keeping the reaction temperature below $5{ }^{\circ} \mathrm{C}$. After $\mathrm{POCl}_{3}$ added completely, the reaction solution was stirred at reflux for $12 \mathrm{~h}$. Then the mixture was poured into a cooled saturated sodium bicarbonate solution, neutralized with odium bicarbonate solution, extracted with dichloromehane and washed with saturated brine. The organic layer separated and dried over anhydrous magnesium sulfite. The crude product was purified using column chromatography with petroleum ether/dichloromethane $(11: 1, \mathrm{v} / \mathrm{v})$ as the eluent. The product was obtained as yellow solids. Yield: $0.8 \mathrm{~g},(84 \%)$. ${ }^{1} \mathrm{H} \mathrm{NMR}(400 \mathrm{MHz}$, $\left.\mathrm{CDCl}_{3}, \mathrm{ppm}\right): \delta=9.89(\mathrm{~s}, 2 \mathrm{H}), 8.39(\mathrm{~s}, 2 \mathrm{H}), 7.79(\mathrm{~d}, \mathrm{~J}=10.1 \mathrm{~Hz}, 4 \mathrm{H}), 7.44(\mathrm{~m}, 4 \mathrm{H}), 4.29(\mathrm{t}, \mathrm{J}=6.6 \mathrm{~Hz}$, $2 \mathrm{H}), 1.87(\mathrm{~s}, 2 \mathrm{H}), 1.30(\mathrm{~m}, 10 \mathrm{H}), 0.85(\mathrm{t}, \mathrm{J}=6.2 \mathrm{~Hz}, 3 \mathrm{H}) .{ }^{13} \mathrm{C} \mathrm{NMR}\left(100 \mathrm{MHz}, \mathrm{CDCl}_{3}\right): \delta 181.57$, $154.67,140.52$, 136.80, 123.97, 122.16, 117.65, 108.74, 42.50, 30.73, 29.90, 28.26, 26.23, 21.56, 13.03 . 


\section{Synthesis of (2E,2'E)-3,3'-(5,5'-(9-octyl-9H-carbazole-3,6-diyl)bis(thiophene-5,2-diyl))bis(2- cyanoacrylic acid) (4)}

A mixture of 5,5'-(9-octyl-9H-carbazole-3,6-diyl)bis(thiophene-2-carbaldehyde) (3) (0.45 g, 0.9 mmol), 2-cyanoacetic acid ( $0.23 \mathrm{~g}, 2.7 \mathrm{mmol})$, ammonium acetate $(0.21 \mathrm{~g}, 2.7 \mathrm{mmol})$ in AcOH (45 $\mathrm{mL}$ ) was stirred for $12 \mathrm{~h}$ at $120{ }^{\circ} \mathrm{C}$ under $\mathrm{N}_{2}$. After it was cooled to room temperature, the reaction mixture was poured into ice water then filtered. The product was obtained as red solid. Yield: $0.47 \mathrm{~g}$ (82 \%). ${ }^{1} \mathrm{H}$ NMR (400 MHz, DMSO) $\delta 8.75(\mathrm{~s}, 2 \mathrm{H}), 8.47(\mathrm{~s}, 2 \mathrm{H}), 8.02(\mathrm{~d}, \mathrm{~J}=3.6 \mathrm{~Hz}, 2 \mathrm{H}), 7.88(\mathrm{~d}, \mathrm{~J}$ $=8.4 \mathrm{~Hz}, 2 \mathrm{H}), 7.80(\mathrm{~d}, \mathrm{~J}=3.8 \mathrm{~Hz}, 2 \mathrm{H}), 7.70(\mathrm{~d}, \mathrm{~J}=8.5 \mathrm{~Hz}, 2 \mathrm{H}), 4.40(\mathrm{~s}, 2 \mathrm{H}), 1.76(\mathrm{~s}, 2 \mathrm{H}), 1.20(\mathrm{~m}$, $11 \mathrm{H}), 0.79(\mathrm{t}, \mathrm{J}=6.6 \mathrm{~Hz}, 3 \mathrm{H}) .{ }^{13} \mathrm{C}$ NMR $(101 \mathrm{MHz}, \mathrm{DMSO}) \delta 164.03,154.80,146.45,141.39$, 133.58,124.94, 124.08, 122.76, 119.01, 116.85, 110.54, 97.44, 42.68, 31.23, 30.73, 28.6, 26.47, 22.08, 13.93.

\section{Conclusions}

In conclusion, the target compound $\mathbf{4}$ has been successfully prepared via the Knevenagel reaction in acceptable yields with a much milder and more efficient route, were designed to construct novel donor-acceptor conjugated organic molecules based on central carbazole units as electronic donors and cyanoacetic acid terminal groups as electronic acceptors.Optical properties, electrical Properties and thermodynamic properties will be studied in future work.

\section{Acknowledgements}

We are grateful for the financial support of the National Natural Science Foundation of China (No. 21462018), the Science Fund of the Technology Office of Jiangxi, China (20122BAB203017 and 20132BBE50024).

\section{Reference}

[1]. Brabec, C.; Sariciftci, N. S.; Gouloumis, A.; et al., J. Mater. Chem. 2003, 13, 700-704.

[2]. Huo, Y. P,; Fang, X. M,; Huang, B. H,; Zhang, K. N. New Progress of Researches in Fluorene Compounds. Chin. J. Org. Chem. 2012, 32, 1169-1185.

[3]. (a) Zhang, X. H.; Chen, B. J.; Lin, X. Q.; Wong, O. Y.; Lee. C. S.; Kwong, H. L.; Lee, S. T.; Wu, S. K. Chem. Mater. 2001, 13, 1565-1569. (b) Li, J. Y.; Liu, D.; Hong, Z. R.; Tong, S. W.; Wang, P. F.; Ma, C. W.; Lengyel, O.; Lee, C. S.; Kwong, H. L.; Lee, S. T. Chem. Mater. 2003, 15, 1486-1490.

[4]. Loi, M. A.; Denk, P.; Hoppe, H.; Neugebauer, H.; Winder, C.; Meissner, D.; Brabec , C.; Sariciftci, N. S.; Gouloumis, A.; Vazquezb, P.; Torresb, T. J. Mater. Chem. 2003, 13, 700-704.

[5]. (a)Li, Y. W.; Guo, Q.; Li, Z. F.; Pei, J. N.; Tian, W. J. Energy Environ. Sci. 2010, 3, 1427. (b)Gustafsson, G.; Gao, Y.; Treacy, G. M.; Klavetter, F.; Colaneri, N.; Heeger, A. J. Nature 1992,357, 477. (c) Roncali, J.; Frere, P.; Blanchard, P.; Bettignies, R.; Turbiez, M.; Roquet, , S.; Leriche, P.; Nicolas, Y. Thin Solid Films 2006, 511/512, 567.

[6]. TANG, Z.; SUN, X.; MIAO, L.; SHEN, H.. Synthesis of a novel donor unit for organic light-emitting materials: 10-octyl-3,7-di(thiophen-2-yl)-10H-phenothiazine. Advanced Materials Research. 2014, 989-994, 284-287.

[7]. TANG, Z.; SUN, X.; YAN, H.; MIAO, L.. Synthesis of a novel D-A type molecule based on carbazole unit for organic light-emitting material applications. Advanced Materials Research. 2014, 989-994, 292-296.

[8]. TANG, Z.; SUN, X.;Zhang, P.. Synthesis and optical properties of D-A type organic molecules based on fluorene and carbazole. Advanced Materials Research. 2015, 1061-1062, 
291-295.

[9]. Chen, C. H.; Tang, C. W. Appl. Phys. Lett. 2001, 79, 3711. (b)Thomas, K. R. J.; Lin, J. T.; Tao, Y. T.; Chuen, C. H. Chem. Mater. 2002, 14, 3852. (c) Goes, M.;Verhoeven, J. W.; Hofstraat, H.; Brunner, K. Chem. Phys. Chem 2003, 4, 349. (d) Kulkarni, A. P.; Tonzola, C. J.; Babel, A.; Jenekhe, S. A. Chem. Mater. 2004,16, 4556. (e) Yan, H.; Lee, P.; Armstrong, N. R.; Graham, A.; Evmenenko, G.A.; Dutta, P.; Marks, T. J. J. Am. Chem. Soc. 2005, 127, 3172.

[10]. Li, Z. Q., M. Siklos, N. Pucher, K. Cicha, A. Ajami, W. Husinsky, A. Rosspeintner, E.Vauthey, G. Gescheidt, J. Stampfl and R. Liska. Synthesis and structure-activity relationship of several aromatic ketone-based two-photon initiators. J. Polym. Sci. A Polym. Chem. 2011, 49, 3688-3699.

[11]. Pucher, N., A. Rosspeintner, V. Satzinger, V. Schmidt, G. Gescheidt, J. Stampfl and R. Liska. Structure-activity relationship in D- $\pi$-A- $\pi$-D-based photoinitiators for the two-photon-induced photopolymerization process. Macromolecules. 2009, 42, 6519-6528.

[12]. Sun, X. B.; Liu, Y. Q; Xu, X. J,; Yang, C.H,; Yu, G,; Chen, S. Y,; Zhao, Z.H,; Qiu, W. F,; Li, Y. F,; Zhu, D. B,; J. Phys. Chem. B, 2005,109, 21.

[13]. He, G. S., L.-S. Tan, Q. D. Zheng and P. N. Prasad. Multiphoton absorbing materials: Molecular designs, syntheses, characterizations, and applications.Chem. Rev. 2008, 108, 1245-1330.

[14]. Zhou H, Zhou F, Tang S, et al. Dyes and Pigments, 2012, 92, 633-641.

[15]. Tacca A, Po R, Caldararo M, et al. Electrochimica acta, 2011, 56, 6638-6653. 